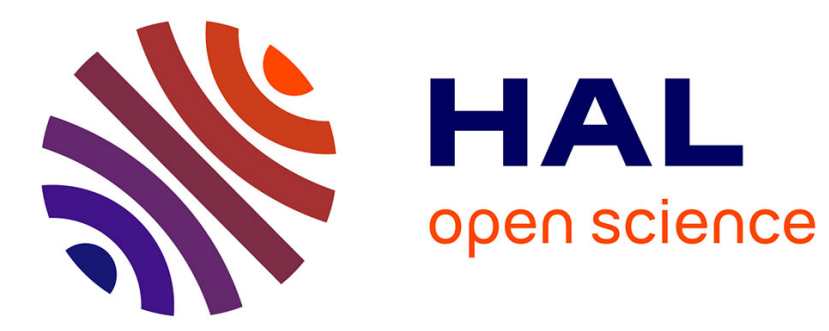

\title{
LQG control for flexible micro-grippers with additional integral action
}

\author{
Yongxin Wu, Héctor Ramirez Estay, Yann Le Gorrec
}

\section{To cite this version:}

Yongxin Wu, Héctor Ramirez Estay, Yann Le Gorrec. LQG control for flexible micro-grippers with additional integral action. Chinese Control conference, Jul 2019, Guangzhou, China. hal-02867756

\section{HAL Id: hal-02867756 \\ https://hal.science/hal-02867756}

Submitted on 15 Jun 2020

HAL is a multi-disciplinary open access archive for the deposit and dissemination of scientific research documents, whether they are published or not. The documents may come from teaching and research institutions in France or abroad, or from public or private research centers.
L'archive ouverte pluridisciplinaire HAL, est destinée au dépôt et à la diffusion de documents scientifiques de niveau recherche, publiés ou non, émanant des établissements d'enseignement et de recherche français ou étrangers, des laboratoires publics ou privés. 


\title{
LQG control for flexible micro-grippers with additional integral action
}

\author{
Yongxin $\mathrm{Wu}^{1}$, Hector Ramirez ${ }^{2}$, Yann Le Gorrec ${ }^{1}$ \\ 1. FEMTO-ST Institute, Univ. Bourgogne Franche-Comté, ENSMM, CNRS, 24 rue Savary, F-25000 Besançon, France. \\ 2. Department of Electronic Engineering, Universidad Tecnica Federico Santa Maria, Avenida Espana 1680, Valparaiso, Chile
}

\begin{abstract}
This paper deals with the stabilizing control design for a class of micro-grippers for DNA manipulation using boundary controlled infinite-dimensional port-Hamiltonian systems. In practical applications, the controllers are implemented as finitedimensional systems actuating at the boundaries of an infinite-dimensional system. The design of the finite-dimensional controllers is still a challenge, especially for hyperbolic PDEs. For this purpose, the LQG balancing reduction method is suitable for the reduced order control design since it considers the closed loop behavior in the reduction procedure. This paper presents the application of this recently proposed method combined with integral action in order to improve the robustness of the closed-loop system. It is shown by means of simulation that the addition of integral action effectively rejects constant perturbations while assuring global closed-loop stability.
\end{abstract}

Key Words: LQG method, port-Hamiltonian system, passive controller, model/controller reduction, integral control

\section{Introduction}

The manipulation of single biological molecules has drawn the attention of researchers in recent years. Several molecule manipulation devices and methods have been proposed such as: magnetic tweezers [1, 2], optical tweezers [3], AFM cantilevers [4] and microfibers [5]. Particular interest is the case of DNA molecule manipulation (Fig. 1).
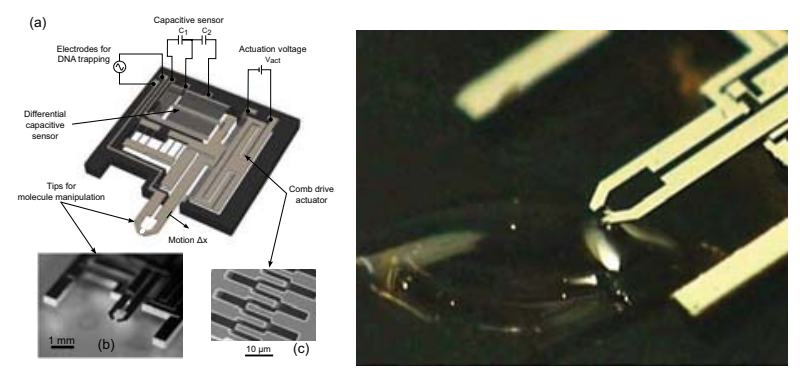

Figure 1: Silicon made micro-grippers

The micro-gripper traps the DNA bundle between two arm tips by using dielectrophoresis and use electrostatic actuation to characterize the mechanical properties of the DNA. It has been shown that micro-grippers are so sensitive to the DNA stiffness variations that they become flexible. Hence current micro-fabrication processes tend to reduce the thickness of the micro-grippers arm in order to improve the sensitivity of the manipulator. It naturally leads to a control problem that can be formulated in terms of partial differential equations (PDEs).

This paper aims to develop a finite-dimensional controller to regulate the angular position of the micro-gripper arm in order to manipulate the DNA bundle by using the port Hamiltonian framework. This framework was first proposed in the context of non linear finite dimensional multi-physical systems $[6,7]$ and generalized to distributed parameters systems in [8]. Due to the geometric properties, the port-

This work was supported by the ANR-DFG (French-German) project INFIDHEM (contract "ANR-16-CE92-0028"), the Bourgogne-Franchecomté Region ANER project $\mathrm{N}^{\circ} 2018 \mathrm{y}-06145$ and the AC3E basal project under the reference FB0008.
Hamiltonian framework is very suitable for the modeling of interconnected systems based on the energy exchange between the sub-system. Thanks to this advantage, we propose to use the port-Hamiltonian framework for modeling and control of flexible micro-grippers in order to grasp the DNA bundle in the liquid phase.

The stabilization problem of infinite-dimensional portHamiltonian systems has been given quite some attention in the last years [9-11]. Even though some results have been validated through numerical simulations of discretized models, it is general hard to approximate PDEs for a large class of infinite-dimensional systems presented by hyperbolic PDEs; since all the eigenvalues are on the imaginary axis, the state variables have the same weight. It is impossible to find the approximated sub variable space by only considering the open loop behavior of the system. As a consequence, in order to find an appropriate approximation scheme, the authors in $[12,13]$ have combined the approximation problem with the LQG control design problem for finite-dimensional port-Hamiltonian systems. Furthermpre, the approach has been generalized to the infinitedimensional port-Hamiltonian systems in [14].

Controllers for infinite-dimensional systems are often based on approximations or discretizations. Additionally micro-gripper usually work at micro or nano scale, they posses flexible arms and are sensitive to noise and disturbances. Thus robust control design method should be considered in order to avoid errors produced in the discretization scheme and due to the additional measurement noise. In practice, integral action is added to the control to counteract the effects of measurement noise. Integral action has been considered within the port-Hamiltonian framework for instance in [15]. The main idea is to impose a portHamiltonian structure in closed-loop in order to guaranteed global asymptotic Lyapunov stability. Recent integral control schemes have been proposed in [16] by using coordinate transformations. The authors in [17] proposed integral action via non passive outputs to deal with nonmatched disturbances. Integral control has also been used to regulate the position by force control for mechanical systems in $[18,19]$. 
These integral control design schemes are proposed for (nonlinear) finite-dimensional port-Hamiltonian systems.

In this paper, we aim to propose a finite-dimensional controller based on the LQG method for a class of micro-gripper in order to preserve the Hamiltonian structure and passivity on the closed loop system. Furthermore an additional integral action will be added in order to guarantee the robustness with respect to perturbations of the controller.

This paper is organized as follows. In Section 2, we recall the port-Hamiltonian formulation of a class of microgripper for DNA manipulation. In Section 3, we use the LQG method to design a finite-dimensional controller with additional integral control action in order to stabilize the infinite port-Hamiltonian system and reject disturbances. We illustrate the proposed controller in Section 4. Finally, conclusions and perspectives are given in Section 5.

\section{Port-Hamiltonian formulation of micro- grippers}

The class of micro-grippers considered in this paper was first presented in [20]. The authors in [21] presented a port-Hamiltonian formulation for a simplified model of the micro-gripper as shown in Fig. 2.

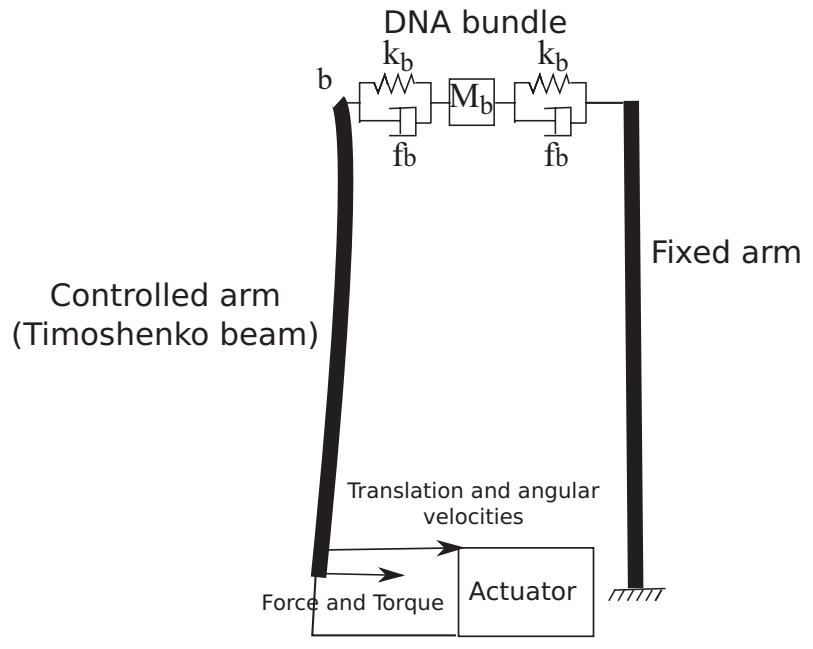

Figure 2: Simplified micro-grippers

The model can be separated into three parts. The first part is a flexible arm that can be modeled using beam theory, more specifically as a Timoshenko beam. The other arm of the micro-gripper is fixed. The second part is the trapped DNA bundle model.The dynamic of the DNA bundle is simplified to a mass-spring-damper mechanical system. The third part is the controller. The three parts are interconnected at the two ends of the gripper's arm in a power conserving way. We introduce the sub-systems in the following.

\subsection{Timoshenko beam modeling of Flexible arm}

Let us first consider the Timoshenko beam described as a boundary controlled port-Hamiltonian system [22, 23]:

$$
\dot{x}=\underbrace{\left(P_{1} \frac{\partial}{\partial z}+P_{0}\right)}_{\mathcal{J}} \mathcal{L} x
$$

with the operator defined by the matrices

$$
\begin{gathered}
\mathcal{L}=\left[\begin{array}{cccc}
K & 0 & 0 & 0 \\
0 & \frac{1}{\rho} & 0 & 0 \\
0 & 0 & E I & 0 \\
0 & 0 & 0 & \frac{1}{I_{\rho}}
\end{array}\right] \\
P_{1}=\left[\begin{array}{llll}
0 & 1 & 0 & 0 \\
1 & 0 & 0 & 0 \\
0 & 0 & 0 & 1 \\
0 & 0 & 1 & 0
\end{array}\right], P_{0}=\left[\begin{array}{cccc}
0 & 0 & 0 & -1 \\
0 & 0 & 0 & 0 \\
0 & 0 & 0 & 0 \\
1 & 0 & 0 & 0
\end{array}\right]
\end{gathered}
$$

with state (energy) variables: the shear displacement $x_{1}=$ $\frac{\partial w}{\partial z}(z, t)-\phi(z, t)$, the transverse momentum distribution $x_{2}=\rho(z) \frac{\partial w}{\partial z}(z, t)$, the angular displacement $x_{3}=\frac{\partial \phi}{\partial z}(z, t)$ and the angular momentum distribution $x_{4}=I_{\rho} \frac{\partial \phi}{\partial z}(z, t)$ for $z \in(a, b), t \geq 0$, where $w(z, t)$ is the transverse displacement and $\phi(z, t)$ is the rotation angle of the beam. The coefficients $\rho, I_{\rho}, E, I$ and $K$ are the mass per unit length, the angular moment of inertia of a cross section, Young's modulus of elasticity, the moment of inertia of a cross section, and the shear modulus respectively, and the state space $X=L_{2}\left(a ; b ; \mathbb{R}^{4}\right)$. The operator $\mathcal{J}=P_{1} \frac{\partial}{\partial z}+P_{0}$ defined by the matrices $P_{1}=P_{1}^{T}$ and $P_{0}=-P_{0}^{T}$ is a first order skew symmetric differential operator acting on the state space $X$. The energy of the beam is expressed in terms of the energy variables,

$$
\begin{aligned}
H & =\frac{1}{2} \int_{a}^{b}\left(K x_{1}^{2}+\frac{1}{\rho} x_{2}^{2}+E I x_{3}^{2}+\frac{1}{I_{\rho}} x_{4}^{2}\right) d z \\
& =\frac{1}{2} \int_{a}^{b} x(z)^{T}(\mathcal{L} x)(z) d z=\frac{1}{2}\|x\|_{\mathcal{L}}^{2}
\end{aligned}
$$

In order to define an extended Dirac structure including the boundary [24], the boundary variables are desired by using integration by part:

$$
\left[\begin{array}{c}
f_{\partial, \mathcal{L} x} \\
e_{\partial, \mathcal{L} x}
\end{array}\right]=\left[\begin{array}{c}
\left(\rho^{-1} x_{2}\right)(b)-\left(\rho^{-1} x_{2}\right)(a) \\
\left(K x_{1}\right)(b)-\left(K x_{1}\right)(a) \\
\left(I_{\rho}^{-1} x_{4}\right)(b)-\left(I_{\rho}^{-1} x_{4}\right)(a) \\
\left(E I x_{3}\right)(b)-\left(E I x_{3}\right)(a) \\
\left(\rho^{-1} x_{2}\right)(b)+\left(\rho^{-1} x_{2}\right)(a) \\
\left(K x_{1}\right)(b)+\left(K x_{1}\right)(a) \\
\left(I_{\rho}^{-1} x_{4}\right)(b)+\left(I_{\rho}^{-1} x_{4}\right)(a) \\
\left(E I x_{3}\right)(b)+\left(E I x_{3}\right)(a)
\end{array}\right]=\left[\begin{array}{c}
v(b)-v(a) \\
F(b)-F(a) \\
w(b)-w(a) \\
T(b)-T(a) \\
v(b)+v(a) \\
F(b)+F(a) \\
w(b)+w(a) \\
T(b)+T(a)
\end{array}\right]
$$

where $F(z), T(z), v(z), w(z)$ are the force, torque, velocity and angular velocity at $z$ point respectively. In additional, we consider some distributed port defined by distributed torques acting on the beam. With the distributed port $\left(\begin{array}{c}f_{d, \mathcal{L} x} \\ e_{d, \mathcal{L} x}\end{array}\right)$, the system becomes:

$$
\begin{aligned}
\dot{x} & =\mathcal{J} \mathcal{L} x+\mathcal{B} e_{d, \mathcal{L} x} \\
f_{d, \mathcal{L} x} & =\mathcal{B}^{*} \mathcal{L} x
\end{aligned}
$$

where the $\mathcal{B}: \mathbb{C}^{i} \mapsto X$ is the distributed input map, $e_{d, \mathcal{L} x} \in \mathbb{C}^{i}$ are the distributed torques applied on the beam, $f_{d, \mathcal{L} x} \in \mathbb{C}^{i}$ are the power conjugated variables of $e_{d, \mathcal{L} x}$, i.e. the angular velocities. Using the mixed finite element semidiscretization method suggested in [25], one obtains a finitedimensional explicit port-Hamiltonian system such as:

$$
\begin{aligned}
\dot{x} & =(J-R) \frac{\partial H}{\partial x}+B u \\
y & =B^{T} \frac{\partial H}{\partial x}
\end{aligned}
$$


where $J=-J^{T}, R=R^{T}>0, H$ is the Hamiltonian function. According to [11], the discretization of the Timoshenko beam model, leads to the following structure matrices:

$$
\begin{gathered}
J_{d}=\underbrace{\left[\begin{array}{cccc}
0 & M & 0 & 0 \\
M^{T} & 0 & 0 & 0 \\
0 & 0 & 0 & M \\
0 & 0 & M^{T} & 0
\end{array}\right]}_{\bar{P}_{1}}+\underbrace{\left[\begin{array}{ccccc}
0 & 0 & B_{1} & 0 \\
0 & 0 & 0 & 0 \\
0 & 0 & 0 & 0 \\
\Phi^{T} & 0 & 0 & 0
\end{array}\right]}_{\bar{P}_{2}} \\
B_{d}=\left[\begin{array}{cccc}
B_{2} & 0 & 0 & 0 \\
0 & 0 & 0 & B_{1} \\
0 & B_{2} & 0 & 0
\end{array}\right]
\end{gathered}
$$

where the sub-matrices are:

$$
\begin{gathered}
M=\left[\begin{array}{ccccc}
-1 & 1 & 0 & \cdots & 0 \\
0 & -1 & 1 & \ddots & \vdots \\
\vdots & \ddots & \ddots & \ddots & 1 \\
0 & \cdots & 0 & 0 & -1
\end{array}\right] \text { with } M \in \mathbb{R}^{N \times N} \\
\Phi=\operatorname{diag}(\beta, \cdots, \beta) \text { with } \Phi \in \mathbb{R}^{N \times N} \\
B_{1}=\left[\begin{array}{c}
0 \\
0_{N-2} \\
1
\end{array}\right] \text { and } B_{2}=\left[\begin{array}{c}
-1 \\
0_{N-2} \\
0
\end{array}\right]
\end{gathered}
$$

where $\beta$ is the distance of the infinitesimal section.

The inputs and outputs of the system are the velocities in translation $v$ and rotation $\omega$ as well as the forces $F$ and torques $T$ at the boundaries $a$ and $b$ :

$$
\begin{aligned}
& u=\left[\begin{array}{llll}
v(b) & \omega(b) & F(a) & T(a)
\end{array}\right]^{T} \\
& =\left[\begin{array}{ll}
u(b) & u(a)
\end{array}\right]^{T} \\
& y=\left[\begin{array}{llll}
F(b) & T(b) & -v(a) & -\omega(a)
\end{array}\right]^{T} \\
& =\left[\begin{array}{ll}
y(b) & y(a)
\end{array}\right]^{T}
\end{aligned}
$$

\subsection{Modeling of DNA bundle}

The DNA bundle can be modeled as a simple finitedimensional port-Hamiltonian system as follow.

$$
\left\{\begin{array}{l}
\dot{v}_{d}=\left(J_{d}-R_{d}\right) \frac{\partial E_{d}}{\partial v_{d}}+g_{d} u_{d} \\
y_{d}=g_{d}^{T} \frac{\partial E_{d}}{\partial v_{d}}+S_{d} u_{d}
\end{array}\right.
$$

where the sub-matrices are: The matrices of DNA bundle are defined as follow:

$$
\begin{gathered}
J_{d}=-J_{d}^{T}=\left[\begin{array}{ccc}
0 & 0 & 1 \\
0 & 0 & 1 \\
-1 & -1 & 0
\end{array}\right], \\
R_{d}=R_{d}^{T}=\left[\begin{array}{ccc}
\frac{1}{f_{1}} & 0 & 0 \\
0 & 0 & 0 \\
0 & 0 & f_{2}
\end{array}\right] \\
g_{d}^{T}=\left[\begin{array}{ccc}
\frac{1}{f_{1}} & 0 & 0 \\
0 & 0 & f_{\theta}
\end{array}\right], \quad S_{d}=\left[\begin{array}{cc}
\frac{1}{f_{1}} & 0 \\
0 & f_{\theta}
\end{array}\right]
\end{gathered}
$$

with the energy of the DNA bundle is given as:

$$
H_{d}=\frac{1}{2}\left(k_{1}\left(x_{c 2}-x_{c 1}\right)^{2}+k_{2} x_{c 2}^{2}+\frac{1}{M}\left(p_{c 2}\right)^{2}\right)
$$

where $M$ is the mass of DNA bundle, $x_{c 1}$ and $x_{c 2}$ are the relative positions of point $b$ and mass $M$ and $p_{c 2}=M \dot{x}_{c 2}$ is its momentum. $k_{1}, k_{2}, f_{1}$ and $f_{2}$ represent the constants of the springs and the viscous dampers of the DNA bundle respectively. $f_{\theta}$ is the rotation damper of the DNA bundle in point $b$.

The inputs of the DNA bundle model are the force and the torque applied on the left side (point $b$ ), which implies the input vector is $u_{d}=\left[\begin{array}{ll}F & T\end{array}\right]^{T}$. The outputs are their power conjugate variables, i. e. the translation velocity and the angular velocity at the same point, which implies that the output vector is $y_{d}=\left[\begin{array}{ll}v & w\end{array}\right]^{T}$.

The gripper arm and the trapped DNA bundle are interconnected at the point $b$. The interconnection relation is

$$
u_{d}=y(b) \quad u(b)=-y_{d}
$$

The physical interpretation of this interconnection law is that when the gripper arm traps the DNA bundle, it applies the force and the torque on the bundle and from the power conserving point of view, the DNA bundle gives back the velocity and angular velocity as the input of the arm at the same point. By using the interconnection relation (18), we get a complete model of this system with total energy being the sum of energy the two sub systems, i.e. $H_{t}=H+H_{d}$.

In the next section, we will discuss the control design method for the micro-gripper model.

\section{Passive LQG controller and additional integral action}

In this section, we shall define a LQG control problem which corresponds to a control by interconnection design problem [26, 27] for linear port-Hamiltonian systems and is suitable for structure preserving reduction of portHamiltonian systems.

\subsection{Port-Hamiltonian system and its LQG control}

First we extend the definition of linear port-Hamiltonian system (PHS) [28, Chap. 6] with some system and measurement noise in order to be able to formulate a LQG problem.

Definition 1. A linear dissipative port-Hamiltonian system (PHS) with state variable $x(t) \in \mathbb{R}^{n}$, input variable $u(t) \in$ $\mathbb{R}^{m}$, output variable $y(t) \in \mathbb{R}^{m}$ and additive system and measurement noises $v, w$ is defined as follows:

$$
\left\{\begin{aligned}
\dot{x} & =(J-R) \frac{\partial H}{\partial x}+B u+v \\
y & =B^{T} \frac{\partial H}{\partial x}+w
\end{aligned}\right.
$$

where $J=-J^{T} \in \mathbb{R}^{n \times n}$ is the skew-symmetric structure matrix, $R=R^{T} \in \mathbb{R}^{n \times n}$ is the symmetric positive dissipation matrix. The total energy of the system is $H=\frac{1}{2} x^{T} Q x$ with $Q=Q^{T} \in \mathbb{R}^{n \times n}$ is the symmetric and positive definite energy matrix. $B \in \mathbb{R}^{n \times m}$ is the input matrix. $\frac{\partial H}{\partial x}=Q x$ are called the co-energy variables. The system's and measurement noises $v, w$ are assumed to be independent white Gaussian processes with the two covariance matrices $Q_{v}$, $R_{w}$

$$
\begin{gathered}
E\left[w(t) w^{T}(t)\right]=R_{w} \delta(t) \quad E\left[v(t) v^{T}(t)\right]=Q_{v} \delta(t), \\
E\left[w(t) v^{T}(t)\right]=0
\end{gathered}
$$

and

$$
R_{w}=R_{w}^{T}>0 \quad Q_{v}=Q_{v}^{T}>0
$$


Remind that the LQG control problem consists in finding a control which minimizes the following cost function:

$$
J_{c}=\lim _{T \rightarrow \infty} E\left[\int_{0}^{\infty}\left(x^{T} \tilde{Q} x+u^{T} \tilde{R} u\right) d t\right]
$$

where $\tilde{Q}$ and $\tilde{R}$ are two weight matrices with $\tilde{Q}=\tilde{Q}^{T}>0$ and $\tilde{R}=\tilde{R}^{T}>0$.

The solution of this problem may be decomposed into the two following steps which we apply to the PHS (19).

- First step: If the pair $\left((J-R) Q, Q_{v}^{1 / 2}\right)$ is stabilizable and the pair $\left((J-R) Q, B^{T} Q\right)$ detectable, one can estimate the state $x$ by the classic Kalman filter equation, i.e.

$$
\dot{\hat{x}}=(J-R) Q \hat{x}+B u+F\left(y-B^{T} Q \hat{x}\right)
$$

with

$$
F=P_{f} Q B R_{w}^{-1}
$$

where $P_{f}$ is the unique solution of the filter Riccati equation

$$
\begin{gathered}
(J-R) Q P_{f}+P_{f} Q(J-R)^{T} \\
-P_{f} Q B R_{w}^{-1} B^{T} Q P_{f}+Q_{v}=0
\end{gathered}
$$

with $P_{f}=P_{f}^{T}>0$

- Second step: If the pair $((J-R) Q, B)$ is stabilizable and the pair $\left((J-R) Q, \widetilde{Q}^{1 / 2}\right)$ detectable, the optimal control is:

$$
u=-K \hat{x}
$$

with

$$
K=\tilde{R}^{-1} B^{T} P_{c}
$$

where $P_{c}$ is the unique solution of control Riccati equation:

$Q(J-R)^{T} P_{c}+P_{c}(J-R) Q-P_{c} B \tilde{R}^{-1} B^{T} P_{c}+\tilde{Q}=0$

with $P_{c}=P_{c}^{T}>0$

Remark 2. Following [29], we call $P_{f}$ and $P_{c}$ the LQG Grammians of the port-Hamiltonian system (19).

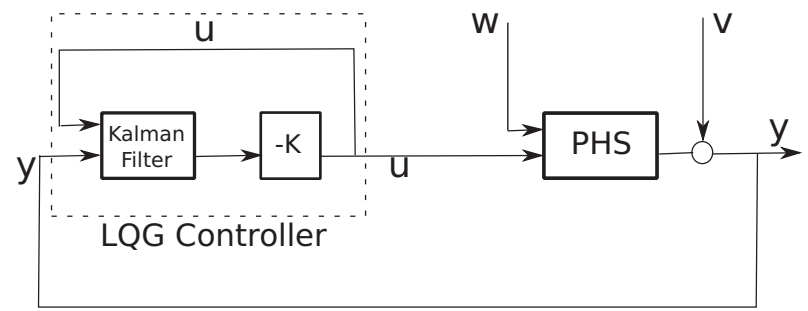

Figure 3: LQG control design to port-Hamiltonian system

The LQG control design is shown in the above figure (Fig. 3). The LQG controller may be expressed as the feedback interconnection of the system (19) with :

$$
\left\{\begin{aligned}
\dot{\hat{x}} & =\left[(J-R) Q-B K-F B^{T} Q\right] \hat{x}+F u_{c} \\
y_{c} & =K \hat{x}
\end{aligned}\right.
$$

As the matrix $Q$ is assumed to be invertible, using the expressions (22) and (25) and defining the matrix

$$
R_{c}=R+B \tilde{R}^{-1} B^{T} P_{c} Q^{-1}+P_{f} Q B R_{w}^{-1} B^{T},
$$

the controller may be written in a quasi-Hamiltonian form:

$$
\left\{\begin{aligned}
\dot{\hat{x}} & =\left(J-R_{c}\right) Q \hat{x}+P_{f} Q B R_{w}^{-1} u_{c} \\
y_{c} & =\left(\tilde{R}^{-1} B^{T} P_{c} Q^{-1}\right) Q \hat{x}
\end{aligned}\right.
$$

In this expression the state matrix is indeed decomposed into the product $\left(J-R_{c}\right) Q$ with the energy matrix $Q$ of the port-Hamiltonian system (19) however the matrix $R_{c}$ is in general neither symmetric nor positive. Furthermore the input and output matrices are not conjugated with respect to the energy. Indeed it is well-known that LQG controller are in general neither stable nor passive.

\subsection{Control by interconnection with LQG control and reduced order controller design}

In order to preserve the passivity and the Hamiltonian structure in the closed loop system using the LQG controller, we shall consider an Hamiltonian structure preserving LQG control design method as follow:

Theorem 3. [13] Denote the LQG Gramians $P_{f}$, solution of the filter Riccati equation (23) and $P_{c}$, solution of the control Riccati equation (26). Consider the LQG problem with the following relation between the covariance matrix $R_{w}$ and the weighting matrix $\tilde{R}$

$$
R_{w}=\tilde{R}
$$

and the relation between the covariance matrix $Q_{v}$ and weighting matrix $\tilde{Q}$ is given by :

$$
Q_{v}=Q^{-1}\left(2 Q J^{T} P_{c}+2 P_{c} J Q+\tilde{Q}\right) Q^{-1}
$$

Then the LQG Gramians satisfy the following relation:

$$
P_{c} Q^{-1}=Q P_{f}
$$

Furthermore, assuming that the port-Hamiltonian system is stable, the control Riccati equation (26) and the filter Riccati equation (23) admit a unique solution, the LQG controller is passive and the closed loop system can be written as the feedback interconnection of the port-Hamiltonian system (19) with the port-Hamiltonian realization of the LQG controller.

Remark 4. The choice of parameter $Q_{v}$ and $\tilde{Q}$ is free. That means that on one hand we can firstly chose the weight matrix $\tilde{Q}$ and then we compute the co-variance matrix $Q_{v}$ as in Theorem 3. On the other hand we can define the co-variance matrix $Q_{v}$, we can obtain the weight matrix $\tilde{Q}$.

In this passive LQG controller, the product of the LQG Grammians is:

$$
P_{f} P_{c}=P_{f} Q P_{f} Q
$$

which permits us to find a balanced base where we can separate the state variables in the sense of closed loop performance. The readers can find all the details about reduction and reduced order LQG control design in [12]. The closed loop port-Hamiltonian system with reduced order LQG con- 
trol is presented as:

$$
\begin{gathered}
\underbrace{\left[\begin{array}{c}
\dot{x} \\
\dot{x}_{c}
\end{array}\right]}_{\dot{x}_{d}}=\underbrace{\left[\begin{array}{cc}
J-R & -B B_{c}^{T} \\
B_{c} B^{T} & J_{c}-R_{c}
\end{array}\right]}_{\left(J_{d}-R_{d}\right)} \underbrace{\left[\begin{array}{c}
\frac{\partial H_{d}}{\partial x_{d}} \\
\frac{\partial H_{d}}{\partial x_{c}}
\end{array}\right]}_{\frac{\partial H_{d}}{\partial x_{d}}}+\underbrace{\left[\begin{array}{c}
B \\
0
\end{array}\right]}_{B_{d}} u \\
y=\underbrace{\left[\begin{array}{ll}
B^{T} & 0
\end{array}\right]}_{B_{d}^{T}} \underbrace{\left[\begin{array}{c}
\frac{\partial H_{d}}{\partial x} \\
\frac{\partial H_{d}}{\partial x_{c}}
\end{array}\right]}_{\frac{\partial H_{d}}{\partial x_{d}}}
\end{gathered}
$$

where the extended state variables $x_{d}^{T}=\left[x^{T}, x_{c}^{T}\right]$, the extended input matrix $B_{d}^{T}=\left[B^{T}, 0\right]$ and the Hamiltonian (total energy) of the closed system

$$
H_{d}=\frac{1}{2} x^{T} Q x+\frac{1}{2} x_{c}^{T} Q_{c} x_{c}
$$

where the matrix $Q_{c}$ is the energy matrix of the reduced order LQG controller.

\subsection{Additional integral action}

In practical applications, integral control is widely used as part of the control systems to improve their robustness since it is quite important to attenuate the effect of measurement noises and model uncertainties. In the framework of port-Hamiltonian systems, [30] propose an extended port controlled Hamiltonian system providing integral action on the passive outputs. In this paper, we show that the extended LQG type controlled port-Hamiltonian system with additional integral action allows to preserve the Hamiltonian structure.

Proposition 5. Consider the the closed loop LQG controlled port-Hamiltonian system (33) with the additional integral action $u=\xi$, where

$$
\xi=-K_{I} \int y d t=-K_{I} \int B_{d}^{T} \frac{\partial H_{d}}{\partial x_{d}} d t
$$

with $K_{I}=K_{I}^{T}>0$. Then all the stability and passivity properties are preserved.

Proof. The closed loop with the integral action (34) takes the port-Hamiltonian form

$$
\left[\begin{array}{c}
\dot{x}_{d} \\
\dot{\xi}
\end{array}\right]=\left[\begin{array}{cc}
J_{d}-R_{d} & B_{d} K_{I} \\
-K_{I} B_{d}^{T} & 0
\end{array}\right]\left[\begin{array}{c}
\frac{\partial H_{e}}{\partial x_{d}} \\
\frac{\partial H_{e}}{\partial \xi}
\end{array}\right]
$$

where

$$
H_{e}=H_{d}\left(x_{d}\right)+\frac{1}{2} \xi^{T} K_{I}^{-1} \xi>0
$$

is a candidate Lyaponov equation to prove the stability of the closed loop system. The power balance equation shows:

$$
\dot{H}_{e}=-\frac{\partial H_{e}^{T}}{\partial x_{d}} R_{d} \frac{\partial H_{e}}{\partial x_{d}}<0
$$

Thus the Lyaponov method can be used to prove the asymptotic stability of the closed loop system with the integral action (35) considering the two above equations (36) and (37).

\section{Numerical results}

In this section, we compare the different control design methods for stabilizing the micro-gripper by simulation. Before the control design procedure, we take 60 infinitesimal subsections of the infinite-dimensional Timoshenko beam to discretize the arm of the micro-gripper and interconnect it with the simplified model of DNA bundle (243 state variables). Then by using the LQG reduction and control design method mentioned in Section 3, we achieve a low order LQG controller (10 state variables for the controller) which can stabilize the full order system. At last, we add the integral action to this reduced order LQG controller in order to improve the robustness of the controller.

In Fig. 4 we compare the step responses of the openloop micro-gripper, the closed loop system with reduced order LQG controller and the LQG controller plus integral action respectively. We measure the angular position of the

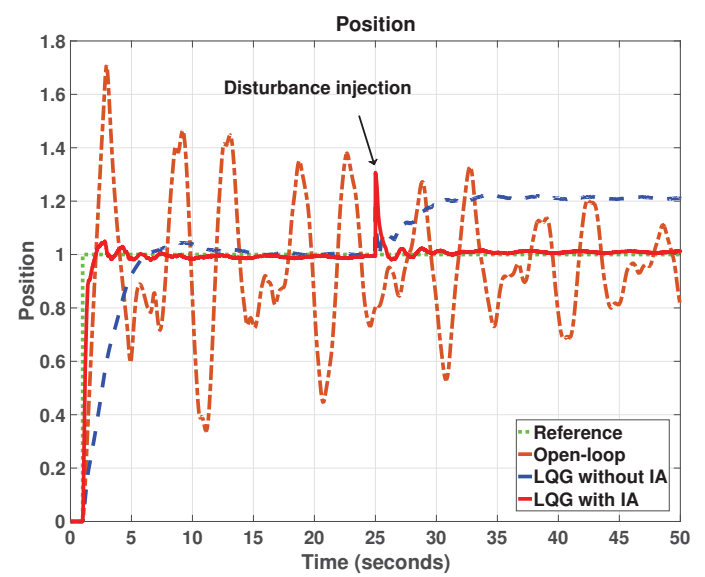

Figure 4: Step responses of the angular positions by using the different controller

DNA manipulator's arm at the contact point with the DNA for an unitary step position reference. The step responses of the open-loop system (orange dashed-dotted curve) is oscillated around the reference position since the system is poorly damped. The closed-loop system with the LQG controller response is shown by the blue dashed curve. We observe that the LQG controller stabilizes the system. However, as well know, due to its weak robustness, the LQG controller cannot reject the constant disturbance which appears at 25 second. An error has been created due to this disturbance. Now we add an integral action in the LQG controlled system as shown in subsection 3.3. The step response has been shown by the red solid curve. One can see on one hand, the response time becomes faster. On the other hand, the disturbance has been rejected using the LQG controller plus an integral action. It shows that the LQG controller with additional integral action improves the robustness of the closed loop system.

\section{Conclusion}

In this paper we considered the control design for a class of DNA manipulation micro-grippers. The port-Hamiltonian approach has been used to model the system as an infinite dimensional system interconnected with a simplified DNA 
bundle model. A finite-dimensional controller has been implemented for this infinite-dimensional system by using the LQG balancing reduction and control design method. However, due to the weak robustness of LQG control design method, we should consider how to guarantee the robustness in the control design. For this purpose, we have added integral action to design a robust controller. The simulation results show the effectiveness of the proposed control design method.

Ongoing work deals with adaptive integral control design for infinite-dimensional port-Hamiltonian systems. In this paper, the results have been proposed based on the discretized model. The stability results have not been proved in the infinite-dimensional case. Hence the theoretical proof for the proposed method will be studied in the future.

\section{References}

[1] F. Amblard, B. Yurke, A. Pargellis, and S. Leibler, "A magnetic manipulator for studying local rheology and micromechanical properties of biological systems," Review of Scientific Instruments, vol. 67, no. 3, pp. 818-827, 1996.

[2] C. Gosse and V. Croquette, "Magnetic tweezers: Micromanipulation and force measurement at the molecular level," Biophysical Journal, vol. 82, no. 6, pp. 3314 - 3329, 2002.

[3] R. Simmons, J. Finer, S. Chu, and J. Spudich, "Quantitative measurements of force and displacement using an optical trap," Biophysical Journal, vol. 70, no. 4, pp. 1813 - 1822, 1996.

[4] E.-L. Florin, V. T. Moy, H. E. Gaub, et al., "Adhesion forces between individual ligand-receptor pairs," ScienceAAAS-Weekly Paper Edition-including Guide to Scientific Information, vol. 264, no. 5157, pp. 415-417, 1994.

[5] A. Ishijima, T. Doi, et al., "Sub-piconewton force fluctuations of actomyocin in vitro," Nature, vol. 352 , no. 6333, p. 301, 1991.

[6] V. Duindam, A. Macchelli, S. Stramigioli, and H. e. Bruyninckx, Modeling and Control of Complex Physical Systems The Port-Hamiltonian Approach. Springer, Sept. 2009. ISBN 978-3-642-03195-3.

[7] B. Maschke and A. van der Schaft, "On the Hamiltonian Formulation of Nonholonomic Mechanical Systems," Reports Mathematical Physics, vol. 34, pp. 225-233, 1994.

[8] A. van der Schaft and B. Maschke, "Hamiltonian Formulation of Distributed Parameter Systems with Boundary Energy Flow," Journal of Geometry and Physics, vol. 42, pp. 166194, 2002.

[9] B. Jacob and H. Zwart, Linear Port-Hamiltonian Systems on Infinite-dimensional Spaces. Operator Theory, Springer Basel, 2012.

[10] H. Ramirez, Y. L. Gorrec, and H. Zwart, "Exponential stabilization of a class of flexible microgrippers using dynamic boundary port Hamiltonian control.," in 52nd IEEE Conference on Decision and Control, CDC'13., (Italy), pp. 1-6, Jan. 2013.

[11] A. Macchelli, "Energy Shaping of Distributed Parameter Port-Hamiltonian Systems Based on Finite Element Approximation," Systems \& Control Letters, vol. 60, pp. 579-589, 2011.

[12] Y. Wu, B. Hamroun, Y. Le Gorrec, and B. Maschke, "Structure preserving reduction of port hamiltonian system using a modified lqg method," in Proceeding of the 33rd Chinese Control Conference (CCC), Nanjing, 2014, pp. 3528-3533, IEEE, 2014.

[13] Y. Wu, B. Hamroun, Y. L. Gorrec, and B. Maschke, "Reduced order lqg control design for port hamiltonian systems," Automatica, vol. 95, pp. 86-92, 2018.

[14] Y. Wu, B. Hamroun, Y. Le Gorrec, and B. Maschke, "Reduced order lqg control design of port hamiltonian systems," IEEE Transaction on Automatic Control, Under review, 2018.

[15] R. Ortega and E. Garcia-Canseco, "Interconnection and damping assignment passivity-based control: A survey," Eur. J Control, vol. 10, pp. 432-450, 2004.

[16] A. Donaire and S. Junco, "On the addition of integral action to port-controlled hamiltonian systems," Automatica, vol. 45, no. 8, pp. 1910 - 1916, 2009.

[17] R. Ortega and E. Garcia-Canseco, "Robust integral control of port-hamiltonian systems: The case of non-passive outputs with unmatched disturbances," Systems \& Control Letters, vol. 61, no. 1, pp. 11 - 17, 2012.

[18] D. A. Dirksz and J. M. A. Scherpen, "Power-based adaptive and integral control of standard mechanical systems," in 49th IEEE Conference on Decision and Control (CDC), pp. 46124617, Dec. 2010.

[19] M. Munoz-Arias, J. M. A. Scherpen, and D. A. Dirksz, "Position control via force feedback for a class of standard mechanical systems in the port-hamiltonian framework," in 52nd IEEE Conference on Decision and Control, pp. 1622-1627, Dec 2013.

[20] M. Boudaoud, Y. Haddab, and Y. Le Gorrec, "Modeling and optimal force control of a nonlinear electrostatic microgripper.," IEEE/ASME Transactions on Mechatronics, vol. 18, pp. 1130-1139, 2013.

[21] H. Ramirez and Y. Le Gorrec, "Boundary port hamiltonian control of a class of nanotweezers," in Control Conference (ECC), 2013 European, pp. 566-571, July 2013.

[22] A. Macchelli and C. Melchiorri, "Modeling and control of the Timoshenko beam. the Distributed Port Hamiltonian approach," SIAM Journal On Control and Optimization, vol. 43, no. 2, pp. 743-767, 2004.

[23] B. Jacob and H. J. Zwart, Linear Port-Hamiltonian Systems on Infinite-dimensional Spaces, vol. 223 of Operator Theory: Advances and Applications. Springer Basel, 2012.

[24] Y. Le Gorrec, H. Zwart, and B. Maschke, "Dirac Structures and Boundary Control Systems Associated with Skewsymmetric Differential Operators," SIMA Journal on Control and Optimization, vol. 44, pp. 1864-1892, 2005.

[25] G. Golo, V. Talasila, A. van der Schaft, and B. Maschke, "Hamiltonian Discretization of Boundary Control Systems," Automatica, vol. 40, pp. 757-771, 2004.

[26] R. Ortega, A. van der Schaft, I. Mareels, and B. Maschke, "Putting energy back in control," IEEE Control Systems Magazine, vol. 21, pp. 18-32, April 2001.

[27] R. Ortega, A. van der Schaft, F. Castanos, and A. Astolfi, "Control by interconnection and standard passivity-based control of port-hamiltonian systems," IEEE Trans. on Automatic Control, vol. 53, no. 11, 2008.

[28] B. Brogliato, R. Lozano, B. Maschke, and O. Egeland, Dissipative Systems Analysis And Control: Theory And Applications. Communications and Control Engineering, Springer, 2007. second edition.

[29] J. Möckel, T. Reis, and T. Stykel, "Linear-quadratic gaussian balancing for model reduction of differential-algebraic systems," Internat. J. Control, vol. 84, pp. 1627-1643, 2011.

[30] R. Ortega and E. Garca-Canseco, "Interconnection and damping assignment passivity-based control: A survey," European Journal of Control, vol. 10, no. 5, pp. 432 - 450, 2004. 